\title{
Thank you to our reviewers in 2015
}

\author{
Jacques Caton ${ }^{1}$ and Hatem Galal Said ${ }^{2, *}$ \\ 1 Clinique Orthopédique Emilia de Vialar, Lyon, France \\ 2 Assiut University Hospital, Assiut, Egypt \\ Published online 2 February 2016
}

The editorial team would like to acknowledge all the reviewers that have accepted and completed a review in 2015 for SICOT-J. With their valuable and constructive comments they have contributed to the quality of the articles published in SICOT-J. The journal relies on their personal effort, sharing their expertise and input and is therefore very grateful.

\section{Amr Abdel Hady}

Nasef Mohamed Nasef Abdelatif

Hisham Abdel-Ghani

Ashraf Abdelkafy

Mohamed El-Sayed Abdel-Wanis

Nariman Abol Oyoun

Ijaz Ahmad

alaa azmi ahmad

Khaled Al Saleh

Nuri Aydin

Ufuk Aydinli

Ahmed Azeem

Laszlo Bucsi

Jacques Caton

Hitendra Doshi

Ziyad El Qirem

Ossama El Shazly

Yasser Elbatrawy

Mohammad El-Sharkawi
Essam ElSherif
Khaled Emara
Mehmet Erdil
Osama Farouk
André Ferreira
Matt Fletcher
Patricia Fucs
Boris Holzapfel
Ashok Johari
Ahmed Kandil
Sherif Khaled
Ahmed Khater
Wael Koptan
Fatih Kucukdurmaz
Anna Kulidjian
Ernest Kwek
Ian Lesley

\author{
Noppachart Limpaphayom \\ François Loisel \\ Keith Luk \\ Sébastien Lustig \\ Mostafa Mahmoud \\ Mahmoud Mahran \\ Oliver Marin Pena \\ Robert Meves \\ Ashraf Moharram \\ Jean-Louis Prudhon \\ Narayan Ramachandran \\ Maximilian Rudert \\ Waleed Riad Saleh \\ Alpaslan Senkoylu \\ Rajasekaran Shanmuganathan \\ Vijay Shetty \\ Mohamed H Sobhy
}

The Editors-in-chief

Jacques Caton Hatem Said

\footnotetext{
*Corresponding author: hatemgalal@yahoo.com 